\title{
Numerical modeling of the equatorial electrojet UT-variation on the basis of the model GSM TIP
}

\author{
M. V. Klimenko ${ }^{1}$, V. V. Klimenko ${ }^{2}$, and V. V. Bryukhanov ${ }^{1}$ \\ ${ }^{1}$ Kaliningrad State Technical University, Kaliningrad, Russia \\ ${ }^{2}$ West Department of IZMIRAN, Kaliningrad, Russia
}

\begin{abstract}
In the presented work the results of numerical modeling of the UT-variation of the equatorial electrojet, executed on the basis of the model GSM TIP are presented, taking into account the dynamo electric fields generated by thermospheric winds in a current-carrying layer of the ionosphere at heights $80-175 \mathrm{~km}$ above a surface of the Earth. To the Global Self-consistent Model of the Thermosphere, Ionosphere and Protonosphere (GSM TIP), developed in WD IZMIRAN, a new block for the calculation of electric fields in the ionosphere has been added. In this block the solution of the three-dimensional equation describing the conservation law of the full current in the Earth's ionosphere is reduced to the solution of the two-dimensional equation by integration along geomagnetic field lines. Calculations of parameters of the near-Earth space plasmas have been executed for quiet equinoctial conditions on 22 March 1987 during the minimum of solar activity.
\end{abstract}

It has been shown, that there is a distinct semidiurnal harmonic in the diurnal behavior of the linear density of the equatorial electrojet with maxima at 23:00 UT and 15:00 UT, as well as with minima at 06:00 UT and 20:00 UT. The greatest and smallest values of the peak intensity of the equatorial electrojet with respect to the diurnal behavior can differ by a factor of two. The longitudinal extent of the area of the equatorial electrojet does hardly show any UT-variation, but the greatest longitudinal extent is at 06 UT. With the growth of the peak intensity of the equatorial electrojet its latitudinal extent also increases $\left(\right.$ on $\left.\sim 5-10^{\circ}\right)$ a little. At the same time the equatorial electrojet in the maxima of intensity has approximately an identical width, whereas in the minima the electrojet is narrow in the morning and wide in the afternoon.

As for the surface density of the equatorial electrojet, its UT-variation is much weaker and equals $\sim 1-3 \mathrm{~A} / \mathrm{km}^{2}$ and the peak intensity is equal $\sim 15-20 \mathrm{~A} / \mathrm{km}^{2}$. The latitudinal extent of the surface density of the equatorial electrojet is maximal at 23:00 UT and 15:00 UT and minimal at 06:00 UT and 20:00 UT.

Correspondence to: M. V. Klimenko

(maksim.klimenko@mail.ru)

\section{Introduction}

The equatorial ionosphere is unique in many respects. Global scale dynamo action, i.e. the generation of currents by electromotive forces due to tidal winds results in the generation of planetary scale east-west electric fields at low latitudes around the geomagnetic equator. These electric fields, in combination with the north-south magnetic fields, cause different geophysical phenomena near the geomagnetic equator. The most important equatorial ionospheric phenomena are: the equatorial ionization anomaly (or Appleton anomaly), the equatorial electrojet, and the generation of plasma density irregularities. An outstanding problem is the cause for the day-to-day variability in the intensity of the electric fields that are responsible for the equatorial anomaly and the electrojet. The enhanced variations of the Earth's magnetic field over the equator were explained by Egedal (1947) to be due to an enhanced east-west current flow in a narrow latitudinal belt $\pm 3^{\circ}$ around the geomagnetic equator. This was later named equatorial electrojet by Chapman (1951). The explanation for the electrojet was offered on the basis of the electrodynamics of a horizontally stratified ionosphere with anisotropic conductivities in a horizontal magnetic field. In a magnetoplasma with mutually perpendicular electric $\boldsymbol{E}$ and magnetic $\boldsymbol{B}$ fields, Pedersen currents flow parallel to $\boldsymbol{E}$ and Hall currents flow perpendicular to both $\boldsymbol{E}$ and $\boldsymbol{B}$. In the presence of the almost non-conducting boundaries above and below the dynamo region $(80-175 \mathrm{~km})$, the flow of Hall currents is inhibited. Under such circumstances, the east-west conductivities are enhanced, increasing the flow of currents, namely, the equatorial electrojet. Cowling (1933), Martyn et al. (1948), Baker and Martyn (1953), and Sugiura and Cain (1966) made important contributions to the explanation of this phenomenon.

Connected with the equatorial electrojet are several phenomena occurring in the equatorial ionosphere during quiet and disturbed geomagnetic conditions, such as equatorial spread $\mathrm{F}$ and equatorial plasma bubbles which influence radio waves propagation in the vicinity of geomagnetic equator.

Published by Copernicus Publications on behalf of the URSI Landesausschuss in der Bundesrepublik Deutschland e.V. 


\section{Statement of the problem and a brief description of the model}

In this work results of numerical modeling of the UTvariation of the equatorial electrojet, executed with the Global Self-consistent Model of the Thermosphere, Ionosphere and Protonosphere (GSM TIP) are presented. The calculations were carried out taking into account only the dynamo electric fields generated by winds of the regular thermospheric circulation, but neglecting thermospheric tides on the bottom boundary of thermosphere at a height of $80 \mathrm{~km}$ and in the current-carrying layer of the ionosphere over the altitude range of 80 to $175 \mathrm{~km}$ above the Earth's surface. The GSM TIP was developed in the West Department of IZMIRAN. For given input data (possibly time dependent) the model calculates the time-dependent global threedimensional structure of temperature, composition $\left(\mathrm{O}_{2}, \mathrm{~N}_{2}\right.$, $\mathrm{O}$ ) and mass velocity vector of the neutral gas; and densities, temperatures and vector velocities of atomic $\left(\mathrm{O}^{+}, \mathrm{H}^{+}\right)$ions, molecular $\left(\mathrm{N}_{2}^{+}, \mathrm{O}_{2}^{+}, \mathrm{NO}^{+}\right)$ions and electrons, and the twodimensional distribution of the electric field potential both of dynamo and magnetospheric origin. Additionally the mismatch of the geographic and geomagnetic Earth's axes is taken into account.

The solution is performed numerically on a global grid with a resolution of $5^{\circ}$ in geomagnetic latitude for the neutral atmospheric and ionospheric equations. The integration of the latter equations is executed along geomagnetic field lines. The geomagnetic field is presented in the model by a tilted centred dipole. Field lines are considered to be open at $L>14.9$, where $L=\frac{r}{R_{E}}$ is the Mcllwain parameter. The longitudinal resolution step is $15^{\circ}$ in a spherical geomagnetic coordinate system. In the vertical dimension, the thermospheric code uses 30 height grid points between 80 and 520 $\mathrm{km}$ altitude above the Earth's surface.

The ionospheric part of the code (F2-region and above) has variable spatial steps along the magnetic field lines from a base altitude $175 \mathrm{~km}$ to a maximum distance of 15 Earth's radii. The paper by Namgaladze et al. (1988) is devoted to the detailed description of the general statement of the problem of modeling parameters of the thermosphereionosphere-protonosphere system as a whole. The statement of the problem is also given in papers by Namgaladze et al. (1991), Korenkov et al. (1998).

To the model a new block of calculation of electric fields in the ionosphere is added (Klimenko et al., 2005, 2006a, b). The modelled physical principles, the mathematical structure of the new block of calculation of electric field and zonal current in the Earth's ionosphere of the model GSM TIP and the used algorithm of the calculations has been described in detail by Klimenko et al. (2006a, b). The distribution of the quasi-stationary large-scale electric field in the Earth's ionosphere is described by the current density conservation law:

$\operatorname{div} \boldsymbol{j}=0$. where $\boldsymbol{j}=\widehat{\sigma} \cdot\left(\boldsymbol{E}+\boldsymbol{V}_{n} \times \boldsymbol{B}\right)=\widehat{\sigma} \cdot \boldsymbol{E}^{\prime}$ is the surface density of the current, $\widehat{\sigma}$ is the ionospheric conductivity tensor, $\boldsymbol{E}$ is the electric field of polarization, $\boldsymbol{V}_{n} \times \boldsymbol{B}$ is the dynamo field, $\boldsymbol{V}_{n}$ is the velocity of the average mass motion of the neutral gas, and $\boldsymbol{B}$ is the geomagnetic field induction.

The three-dimensional Eq. (1) was reduced to twodimensional integration over the height of the currentconducting layer. The transition to the two-dimensional equation is performed by taking the integral along the geomagnetic field lines assuming that the electric field is constant in the current-conducting ionospheric layer along these field lines, which are expected equipotential. Such an approach to modeling the electric field in the Earth's ionosphere has long been known and is used by many researchers. This approach has been described in many monographs and manuals, e.g., in (Gurevich et al., 1976; Richmond, 1982; Volland, 1984; Singh and Cole, 1987; Heelis, 2004).

Assuming that the geomagnetic field is a dipole, we introduce the dipole (dipolar) coordinate system $(q, v, u)$, where:

$q=\rho^{2} \cdot \cos \Theta, \quad v=\Lambda, \quad u=\rho \cdot \sin ^{2} \Theta$.

Here $\rho=\frac{R_{E}}{r}=\frac{R_{E}}{R_{E}+h}, R_{E}$ is the Earth's radius, $h$ is the height above the Earth's surface, $(r, \Theta, \Lambda)$ is a spherical geomagnetic coordinate system, $r$ is the radius vector, $\Theta$ is the geomagnetic colatitude (polar angle), and $\Lambda$ is the geomagnetic longitude. The conductivity tensor shape in the dipole (dipolar) and spherical geomagnetic coordinate systems can be found, e.g., in (Gurevich et al., 1976).

In the dipole (dipolar) coordinate system $(q, v, u)$, the equation of current density conservation in the Earth's ionosphere Eq. (1) has the form:

$$
\begin{aligned}
& \frac{1}{h_{q} \cdot h_{v} \cdot h_{u}} \cdot\left(\frac { \partial } { \partial v } \left(h _ { q } \cdot h _ { u } \cdot \left(\sigma_{P} \cdot E_{v}+\sigma_{H} \cdot E_{u}+\right.\right.\right. \\
& \left.\left.+\left(\sigma_{P} \cdot V_{n u}-\sigma_{H} \cdot V_{n v}\right) \cdot B\right)\right)+\frac{\partial}{\partial u}\left(h _ { q } \cdot h _ { v } \cdot \left(\sigma_{P} \cdot E_{u}-\right.\right. \\
& \left.\left.\left.-\sigma_{H} \cdot E_{v}-\left(\sigma_{P} \cdot V_{n v}+\sigma_{H} \cdot V_{n u}\right) \cdot B\right)\right)\right)=0,
\end{aligned}
$$

where $h_{q}=\frac{R_{E}}{\rho^{3} \cdot k}, h_{v}=\frac{R_{E}}{\rho} \cdot \sin \Theta$, and $h_{u}=\frac{R_{E}}{\rho^{2} \cdot k \cdot \sin \Theta}$ are the Lame coefficients, and $k=\sqrt{1+3 \cdot \cos ^{2} \Theta}$

If the electric field potential in the Earth's ionosphere is taken into account, we obtain:

$$
\begin{aligned}
& \frac{\partial}{\partial u}\left(\frac{h_{q} \cdot h_{v}}{h_{u}} \cdot \sigma_{P} \cdot \frac{\partial \Phi}{\partial u}\right)+\frac{\partial}{\partial v}\left(h_{q} \cdot \sigma_{H}\right) \cdot \\
& \frac{\partial \Phi}{\partial u}+\frac{\partial}{\partial v}\left(\frac{h_{q} \cdot h_{u}}{h_{v}} \cdot \sigma_{P} \cdot \frac{\partial \Phi}{\partial v}\right)- \\
& -\frac{\partial}{\partial u}\left(h_{q} \cdot \sigma_{H}\right) \cdot \frac{\partial \Phi}{\partial v}=\frac{\partial}{\partial v}\left(h_{q} \cdot h_{u} \cdot\left(\sigma_{P} \cdot V_{n u}-\sigma_{H} \cdot V_{n v}\right) \cdot B\right)- \\
& -\frac{\partial}{\partial u}\left(h_{q} \cdot h_{v} \cdot\left(\sigma_{P} \cdot V_{n v}+\sigma_{H} \cdot V_{n u}\right) \cdot B\right)
\end{aligned}
$$


where $\Phi$ is the electric field potential; $B=|\boldsymbol{B}|, \sigma_{P}$ and $\sigma_{H}$ are the Pedersen and Hall conductivities of the ionosphere, the expressions of which can be found in (Gershman, 1974; Heelis, 2004).

Let us integrate Eq. (2) along the field line segment in the current-conducting layer from $q_{1}$ to $q_{2}$. If the field line completely lies in the ionospheric current-conducting layer, the integration is performed from the bottom of this line in the given hemisphere to the top.

$\frac{\partial}{\partial u} \int_{q_{1}}^{q_{2}} \frac{h_{q} \cdot h_{v}}{h_{u}} \cdot \sigma_{P} \cdot \frac{\partial \Phi}{\partial u} \cdot d q+\int_{q_{1}}^{q_{2}} \frac{\partial}{\partial v}\left(h_{q} \cdot \sigma_{H}\right) \cdot \frac{\partial \Phi}{\partial u} \cdot d q+$ $+\frac{\partial}{\partial v} \int_{q_{1}}^{q_{2}} \frac{h_{q} \cdot h_{u}}{h_{v}} \cdot \sigma_{P} \cdot \frac{\partial \Phi}{\partial v} \cdot d q-\int_{q_{1}}^{q_{2}} \frac{\partial}{\partial u}\left(h_{q} \cdot \sigma_{H}\right) \cdot \frac{\partial \Phi}{\partial v} \cdot d q=\psi$,

where

$$
\begin{aligned}
& \psi=\frac{1}{R_{E}^{3}} \cdot \frac{\partial}{\partial v} \int_{q_{1}}^{q_{2}}\left(\sigma_{P} \cdot V_{n u}-\sigma_{H} \cdot V_{n v}\right) \cdot B \\
& \frac{r^{5}}{\sin \Theta \cdot\left(1+3 \cdot \cos ^{2} \Theta\right)} \cdot d q-
\end{aligned}
$$$$
-\frac{1}{R_{E}^{2}} \cdot \frac{\partial}{\partial u} \int_{q_{1}}^{q_{2}}\left(\sigma_{P} \cdot V_{n v}+\sigma_{H} \cdot V_{n u}\right) \cdot B \cdot \frac{r^{4} \cdot \sin \Theta}{\sqrt{1+3 \cdot \cos ^{2} \Theta}} \cdot d q
$$

Assuming that inflowing and outflowing currents are absent at the lower boundary of the ionospheric current conducting layer at a height of $80 \mathrm{~km}$, we have the boundary condition for the three-dimensional modeling Eq. (1): $j_{q}=0$ (everywhere except at the equator) and $j_{u}=0$ (at the equator) at a height of $h=80 \mathrm{~km}$, which is used as the lower limit during the integration over the thickness of the ionospheric currentconducting layer.

We now determine the zonal electrojet as a zonal current surface density integrated over the thickness of the ionospheric current-conducting layer, i.e., as a zonal current linear density. In this case the positive and negative signs of the zonal electrojet will correspond to the direction toward east and west, respectively.

In the model where the integration is performed along the geomagnetic field lines, the zonal electrojet is calculated using the formula:

$J_{v}=\sum_{P} \cdot E_{v}+\sum_{H} \cdot E_{u}+\int_{q_{1}}^{q_{2}} B\left(\sigma_{P} \cdot V_{n u}-\sigma_{H} \cdot V_{n v}\right) \cdot h_{q} \cdot d q$,

where $\sum_{P}=\int_{q_{1}}^{q_{2}} \sigma_{P} \cdot h_{q} \cdot d q ; \sum_{H}=\int_{q_{1}}^{q_{2}} \sigma_{H} \cdot h_{q} \cdot d q$

In papers by Klimenko et al. (2006a, b) the modelled physical principles and the mathematical structure of the new

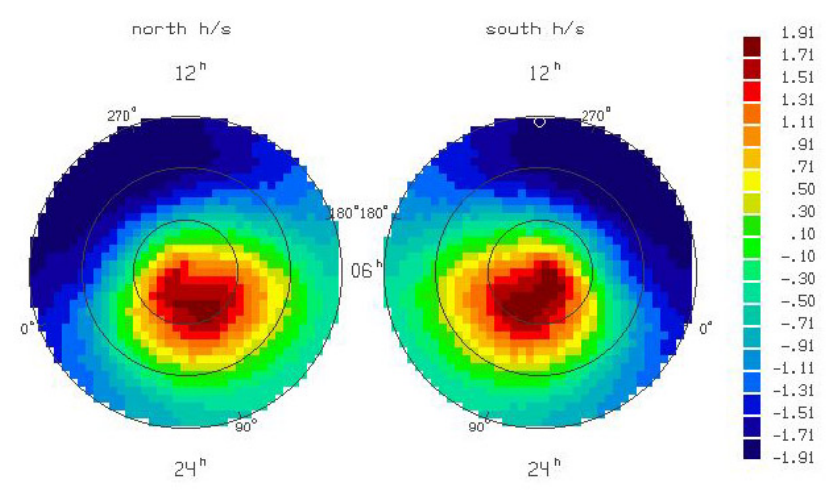

Fig. 1. Dynamo electric field potential distribution in polar geomagnetic coordinate system (latitude-longitude) obtained in the model GSM TIP for 00:00 UT.

block of calculation of electric field and zonal current in the Earth's ionosphere of the model GSM TIP, and the used algorithm of the calculations are explained in every detail.

The equatorial electrojet is essentially a LT (local time) phenomenon with the UT component resulting from longitudinal differences arising from the differences in conductivity due to various factors, e.g. the fact that the dipole axis and the geographic axis do not coincide, or that the ambient magnetic field has a non-dipolar component, or that the magnetospheric sources are contributing to the electric fields or conductivity. From all listed factors in this work there is only the first factor taken into account, namely, that the dipole axis and the geographic axis do not coincide. The second and the third of the above factors are neglected here. Firstly, in the model GSM TIP the dipole approximation of the ambient geomagnetic field is used, therefore a non-dipol component naturally is neglected. Secondly, all calculations in this work were carried out taking into account the dynamo field generated by thermospheric winds only. Therefore the contribution of electric fields of magnetospheric convection is absent.

The inclusion of the new block of the electric field calculation in the model GSM TIP allows us to investigate the equatorial ionosphere. The calculations were carried out for quiet equinox conditions on 22.03.1987 during solar activity minimum $\left(\mathrm{F}_{10.7}=76\right)$.

\section{Calculation results and their discussion}

Figure 1 shows the global distribution of the dynamo electric tem (latitude-longitude), calculated on the basis of GSM TIP for 00:00 UT. The geomagnetic latitudes are shown by circles with steps of $30^{\circ}$ from the geomagnetic pole to the geomagnetic equator for both hemispheres. Numbers on the equatorial circle denote the positions of longitudinal geomagnetic 


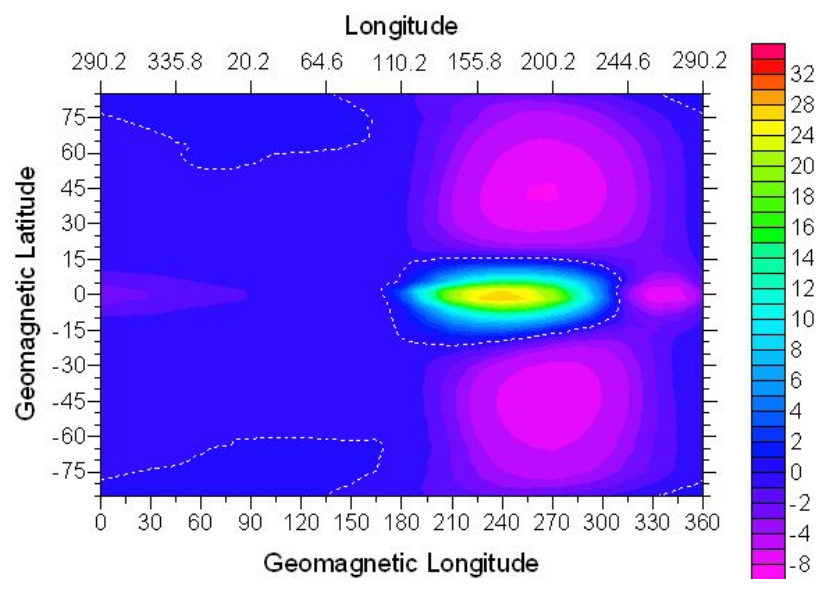

Fig. 2. Zonal current linear density obtained in the model GSM TIP in $\mathrm{A} / \mathrm{km}$ for 23:00 UT.

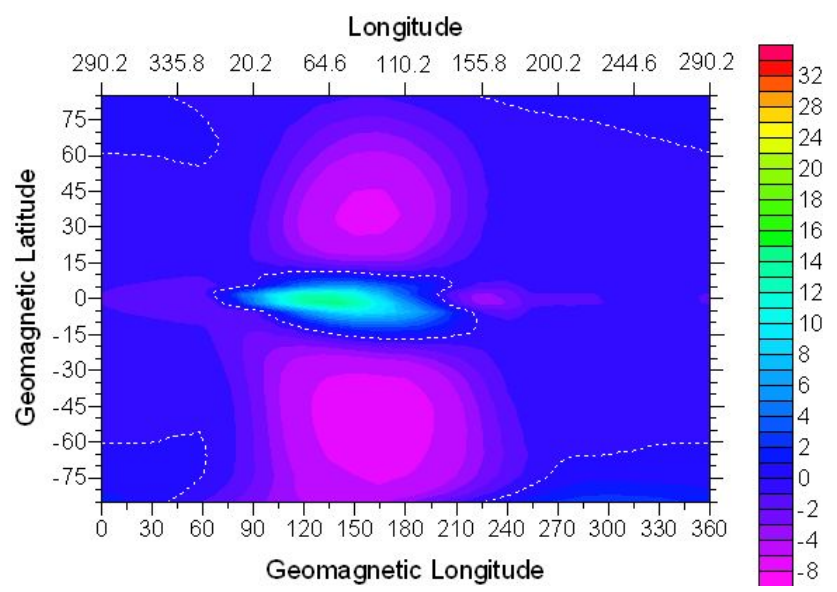

Fig. 3. Zonal current linear density obtained in the model GSM TIP in $\mathrm{A} / \mathrm{km}$ for 06:00 UT.

meridians $0^{\circ}, 90^{\circ}, 180^{\circ}$ and $270^{\circ}$. Also the time in a SolarMagnetospheric coordinate system is shown.

Figures. 2-5 show the global distributions of zonal current linear density, obtained with the model GSM TIP for 23:00 UT, 06:00 UT, 15:00 UT and 20:00 UT in a Cartesian geomagnetic coordinate system (longitude-latitude). One can see that the maximal intensity of the zonal current linear density has maxima at 23:00 UT and 15:00 UT and minima at 06:00 UT and 20:00 UT. The greatest and smallest values of the equatorial electrojet maximal intensity during the day can differ by a factor of two. The longitudinal extent of the equatorial electrojet area does hardly reveal any UTvariation, although it is possible to note the greatest longitudinal extent at 06:00 UT. With amplification of the equatorial electrojet maximal intensity, its latitudinal extent slightly increases also (about $5-10^{\circ}$ ). At the same time, the equatorial electrojet in its maxima of intensity has approximately an

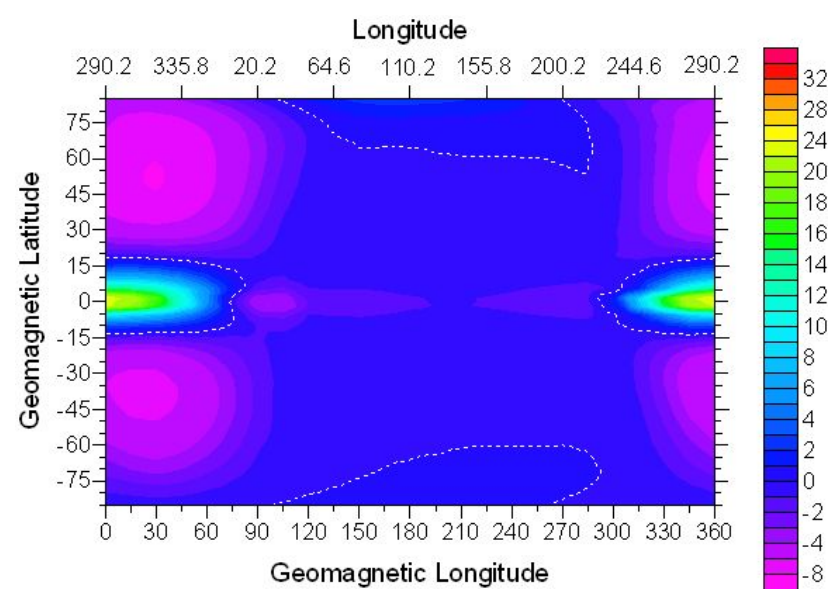

Fig. 4. Zonal current linear density obtained in the model GSM TIP in $\mathrm{A} / \mathrm{km}$ for 15:00 UT.

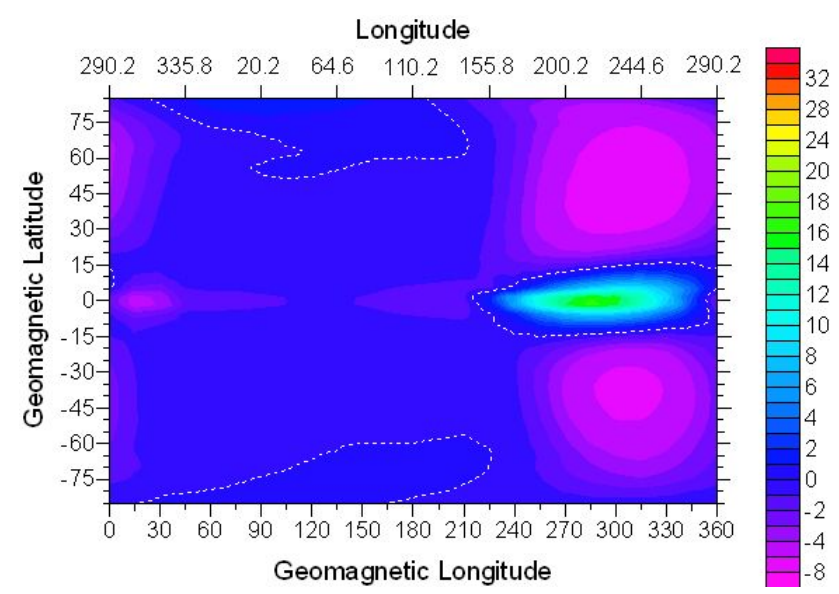

Fig. 5. Zonal current linear density obtained in the model GSM TIP in $\mathrm{A} / \mathrm{km}$ for 20:00 UT.

identical width, and in the minima of intensity the electrojet is narrow in the morning and wide in the afternoon.

Under symmetrical conditions in the Northern and Southern hemisphere the current at the upper boundary of the current-conducting layer should vanish. In reality, an asymmetry is always observed between the hemispheres, since the conductivities and thermospheric circulation are different on both sides of the geomagnetic equator. This asymmetry originates even during equinoctial conditions, because the axes of the Earth rotation and the geomagnetic dipole do not coincide. Therefore, the geomagnetic field lines remain on equipotential (due to the high field-aligned conductivity along these lines) only when currents are generated along the closed geomagnetic field lines. These field aligned currents almost instantaneously compensate the originating potential drop between the hemispheres. On open geomagnetic field lines, these currents are absent due to the absence of any 
relation between the hemispheres.

The majority of researchers speak now only about a longitudinal variation of various parameters of the near-Earth environment, including the equatorial electrojet, forgetting about UT-variation or identifying it with a longitudinal variation. We consider this as incorrect. Really, in an experiment it is very difficult, if at all possibly, to separate a longitudinal variation from a UT-variation. However it is very easy in numerical modeling.

Let us consider the sources of these variations. Originally it is supposed that a UT variation of the ionospheric parameters are connected with the mismatch of the axis of the geomagnetic dipole with the geographic axis of the Earth rotation. For any existence of longitudinal variations the presence of longitudinal sources is necessary. These sources must be located in well-defined places on the Earth's surface. For example, the South-Atlantic anomaly is a source of longitudinal variations. This source is connected with the presence of multipole components in the geomagnetic field. UT and longitude are independent variables. We can expand every function in series of these two independent variables. Herewith one term of the decomposition in the series will depend on longitude only, the other will depend on UT only, but the third (the cross terms of the decomposition) will depend on both variables. The UT variation will be described with the terms of the decomposition, depending on UT. The longitudinal variation will be described with the terms, depending on longitude. But cross terms will describe a dependency on both UT, and longitude. Most likely, the contribution of these terms will be small in comparison with the contribution of the main terms, describing longitudinal and UT variation.

In our model the geomagnetic field is approximated by a central dipole, the axis of which does not coincide with the geographical axis of Earth rotation. In this approximation of a geomagnetic field, there is no non-dipole component. Therefore the visible reasons for an existence of longitudinal variations are absent, while the mechanism of formation of a UT-variations is present. Hence, we shall speak about UT-variations, discussing results of the calculations. Also we shall speak about longitudinal variations, discussing experimental data in which UT and longitudinal variations are contained, since in this case we deal with real data.

The equatorial electrojet is known to peak around 11:0012:00 LT. In our calculation results the maximum intensity of the equatorial electrojet appears around $\Lambda=240^{\circ}$ (11:00 LT) for 23.00 UT, around $\Lambda=130^{\circ}$ (10:40 LT) for 06:00 UT, around $\Lambda=0^{\circ}$ (11:00 LT) for 15:00 UT, and around $\Lambda=280^{\circ}$ (10:20 LT) for 20:00 UT. As for a displacement of the maximum of the electrojet into the morning sector of LT in comparison with real experimental data, this can be caused by neglecting tides on the bottom boundary of thermosphere and by neglecting the electric field of magnetospheric convection in our calculations.

In papers by Lühr et al. (2004), Manoj et al. (2006), Le Mouel et al. (2006) observational data of the equatorial elec- trojet obtained with the CHAMP satellite were analyzed. The analysis of data has shown, that the equatorial electrojet represents a narrow formation on the geomagnetic equator. The width of the equatorial electrojet equals about $2000 \mathrm{~km}$ in the day-time ionosphere. At the same time the maximal intensity of the equatorial electrojet was estimated by Lühr et al. (2004) as $0.15 \mathrm{~A} / \mathrm{m}$ on the average, whereas Manoj et al. (2006) obtained $0.04 \mathrm{~A} / \mathrm{m}$ for the same value. The data of Manoj et al. (2006) correspond to a lower level of solar activity.

Comparing these data with results of our calculations, we can state their satisfactory consent in position and spatial size of the equatorial electrojet. In our calculations the width of the electrojet along latitude turns out a little greater, than in the experiment. This can be explained with the absence of the electric field of magnetospheric convection and the absence of thermospheric tides in our calculations which could lead to a modification of the spatial distribution of the zonal current in the Earth's ionosphere or with the rough spatial grid in our model. As for the maximal intensity of the equatorial electrojet in our calculations, its value of $35 \mathrm{~A} / \mathrm{km}$ is very close to the measurements of Manoj et al. (2006).

A comparison of results of our model calculations with experimental data by Le Mouel et al. (2006) has shown, that:

1. Locations of calculated and observed electrojet at 06:00 UT, 08:00 UT and 22:00 UT practically coincide;

2. While in the experiment the longitudinal extent of the equatorial electrojet depends on UT and is minimal for the southern hemisphere, in the results of the model calculations the longitudinal extent of the equatorial electrojet is constant. This indicates that in the experiment there are sources of longitudinal and of UT variations of the equatorial electrojet (a real geomagnetic field with all features, including the South-Atlantic magnetic anomaly with which the minimal longitudinal extent of the equatorial electrojet in the southern hemisphere is possibly connected). In the model there are only sources of UT variation (dipole geomagnetic field). From this it is possible to draw the conclusion that the variability of the longitudinal extent of the equatorial electrojet is the consequence of a longitudinal variation;

3. In model calculations the counter electrojet is formed both in the morning and in the evening, but in the evening the counter electrojet is much stronger than in the morning. In the experiment the counter electrojet is formed only in the morning. This fact can be explained by the absence of thermospheric tides in the presented calculations.

Figure 6 shows the diurnal behavior of the maximal intensity of the equatorial electrojet. The plot in Fig. 6 has been obtained by a selection of the maximal values of the equatorial electrojet for each calculated UT time within the day, 


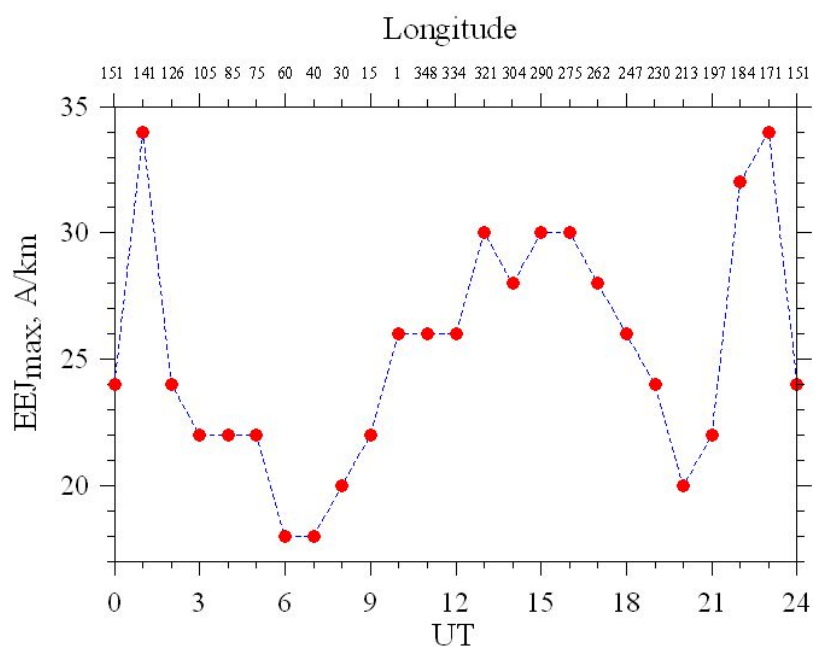

Fig. 6. Diurnal behavior of the maximal intensity of the equatorial electrojet obtained in the model GSM TIP in A/km.

therefore each point on the abscissa of this plot corresponds to conditions close to noon for various geomagnetic longitudes at the geomagnetic equator. It is shown, that there is a precise semidiurnal harmonic in the diurnal behavior of the equatorial electrojet linear density with maxima at 23:00 UT and 15:00 UT and with minima at 06:00 UT and 20:00 UT.

In investigations by Ivers et al. (2003), Doumouya and Cohen (2004), and Le Mouel et al. (2006) obtained on the basis of observations of the equatorial electrojet with the CHAMP and Ørsted satellites, it was shown that the variability of the equatorial electrojet depends on time and longitude.

Ivers et al. (2003) and Le Mouel et al. (2006) have shown the existence of a quarter-diurnal (six-hour) harmonic in the equatorial electrojet, i.e. the presence of a UT or longitudinal behavior of the equatorial electrojet with four maxima and accordingly four minima. The maximal intensity of the equatorial electrojet in our calculations shows a semidiurnal harmonic behavior on UT. We used the geographical longitudes of the maxima from Ivers et al. (2003) in geomagnetic longitude at the geomagnetic equator. Then we recalculated these longitudes in UT which correspond to noon conditions in points: $\lambda=0^{\circ}-30^{\circ} \mathrm{E}$ corresponding to $\Lambda=85^{\circ}$ or 11:00 UT; $\lambda=90^{\circ}-120^{\circ} \mathrm{E}$ corresponding to $\Lambda=175^{\circ}$, or $05: 00 \mathrm{UT}$; $\lambda=180^{\circ}-220^{\circ} \mathrm{E}$ corresponding to $\Lambda=270^{\circ}$, or 22:00 UT; $\lambda=260^{\circ}-290^{\circ} \mathrm{E}$ corresponding to $\Lambda=345^{\circ}$, or 17:00 UT. Thus we obtain a coincidence of maxima of the equatorial electrojet in the longitudinal ranges $\lambda=180^{\circ}-220^{\circ} \mathrm{E}$ (in our calculations 23:00 UT, and in experiment 22:00 UT) and $\lambda$ $=260^{\circ}-290^{\circ} \mathrm{E}$ (in our calculations 15:00 UT, and in experiment 17:00`UT).

Le Mouel et al. (2006) have shown that the longitudinal behavior of the maximal intensity of the equatorial electrojet has four maxima and four minima. Three of the four maxima coincide with the maxima in the paper by Ivers et al. (2003).

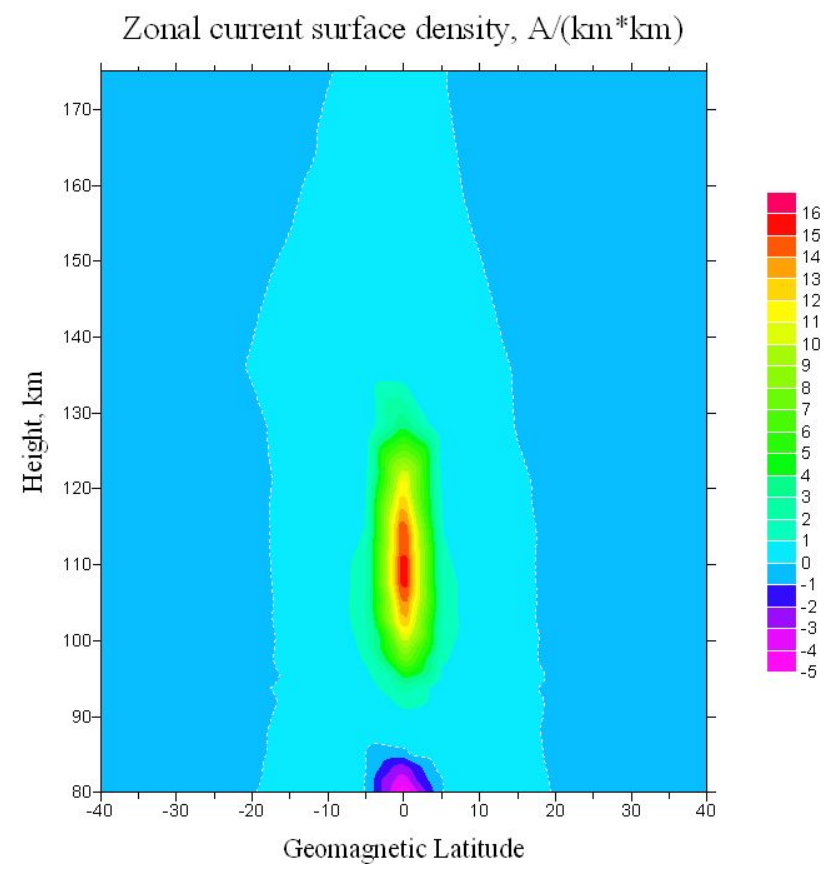

Fig. 7. Zonal current surface density obtained in the model GSM TIP in $\mathrm{A} / \mathrm{km}^{2}$ for 23:00 UT.

From the papers by Doumouya and Cohen (2004) it is possible to pick out six extrema in the longitudinal behavior of the intensity of the equatorial electrojet. Three maxima and three minima from their work coincide with three maxima and three minima in the work by Le Mouel et al. (2006).

According to data from Doumouya and Cohen (2004) and Le Mouel et al. (2006) the longitudinal variation of the equatorial electrojet lays in the range 1.7-2.3 and 2.3-3.2, correspondingly. Figure 6 shows, that the magnitude of the UT variation of the equatorial electrojet equals approximately 2 in our calculations. We obtained this magnitude for quiet geomagnetic conditions during a spring equinox under a minimum of solar activity.

Figures 7-10 show the distributions of zonal current surface density, obtained with the model GSM TIP for 23:00 UT, 06:00 UT, 15:00 UT and 20:00 UT in a Cartesian geomagnetic coordinate system (longitude-altitude). One may notice that its UT-variation is much weaker and equals $\sim 1-3 \mathrm{~A} / \mathrm{km}^{2}$ and herewith the maximal intensity is $\sim 15-$ $20 \mathrm{~A} / \mathrm{km}^{2}$. At the same time the latitudinal extent of the equatorial electrojet surface density is maximal at 23:00 UT and 15:00 UT and minimal at 06:00 UT and 20:00 UT.

A comparison of experimental data and results of model calculations by other authors has been made by Klimenko et al. (2006a). Comparison with simulation results of Richmond (1989) and Stening (1985) and with the experimental data from Stening (1985) has revealed a satisfactory consent for the vertical structure of the zonal current in the equatorial ionosphere. 


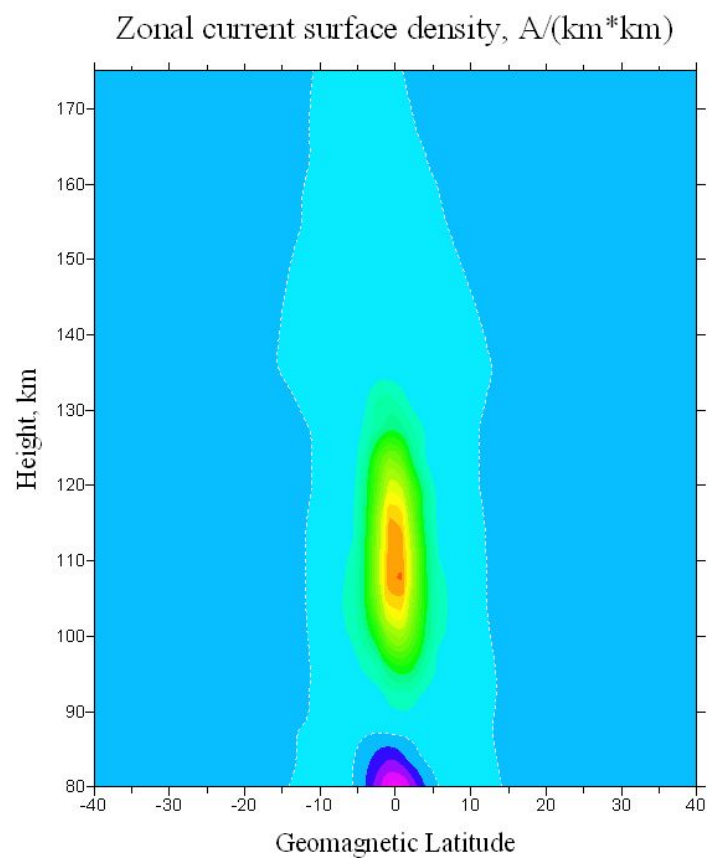

Fig. 8. Zonal current surface density obtained in the model GSM TIP in $\mathrm{A} / \mathrm{km}^{2}$ for 06:00 UT.

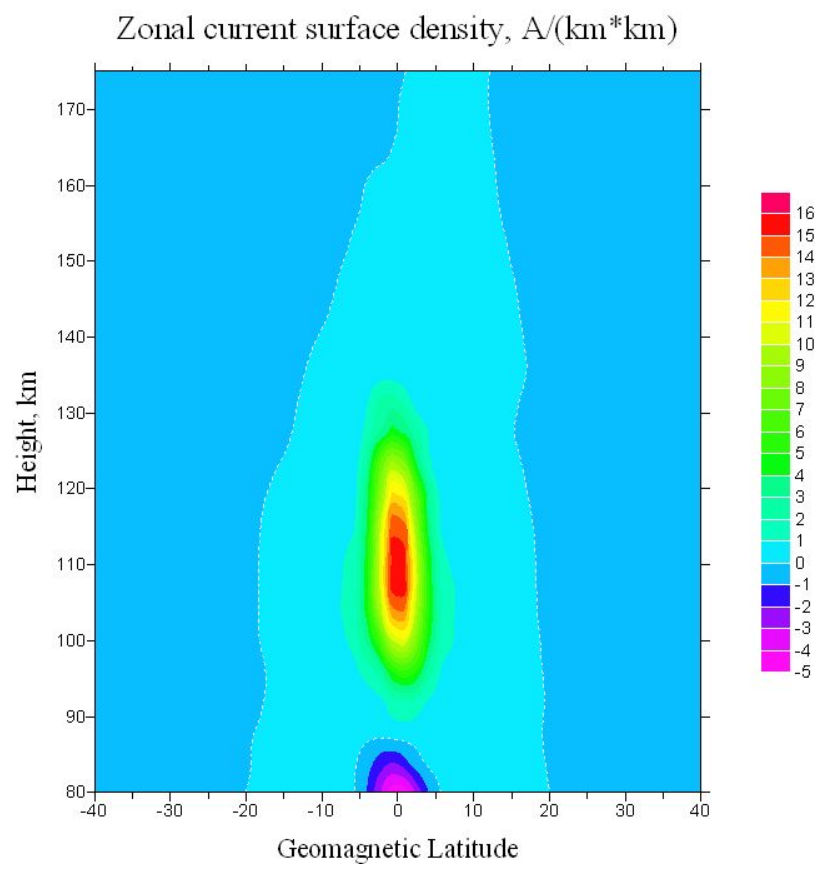

Fig. 9. Zonal current surface density obtained in the model GSM TIP in $\mathrm{A} / \mathrm{km}^{2}$ for 15:00 UT.

\section{Summary}

Numerical calculations have shown, that there is a semidiurnal harmonic in the diurnal behavior of the maximal inten-

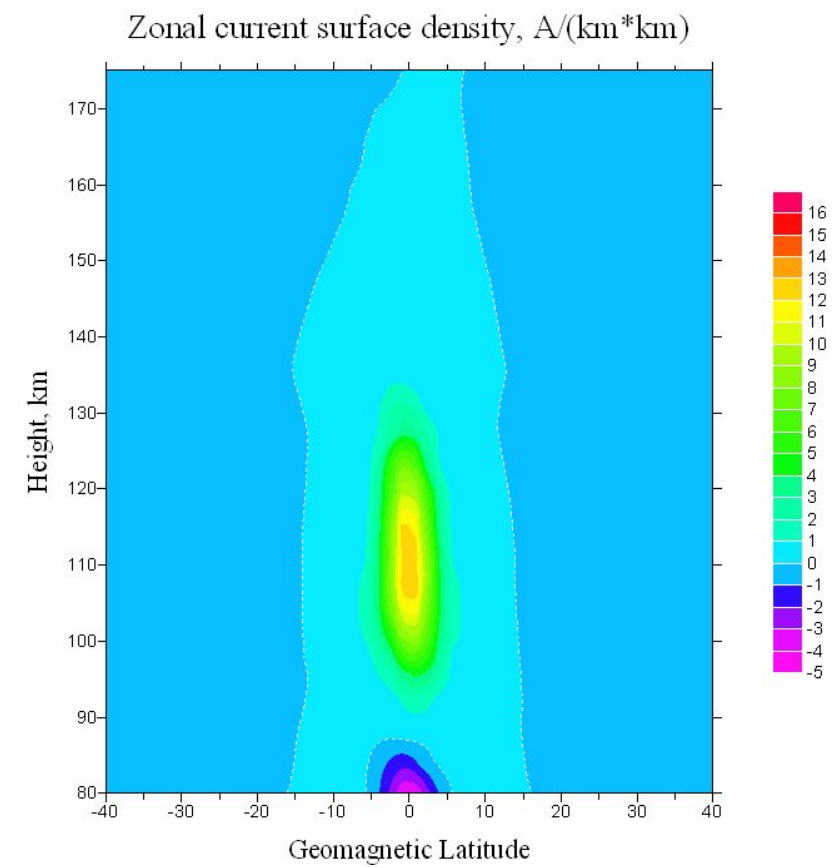

Fig. 10. Zonal current surface density obtained in the model GSM TIP in $\mathrm{A} / \mathrm{km}^{2}$ for 20:00 UT.

sity of the equatorial electrojet with maxima at 23:00 UT and 15:00 UT and with minima at 06:00 UT and 20:00 UT. The greatest and smallest values of its maximal intensity in the diurnal behavior can differ by a factor of two. The longitudinal extent of the area of the equatorial electrojet practically does not show any UT-variation. The variability of the longitudinal extent of the equatorial electrojet with respect to UT, which exists in experiment, is a longitudinal variation but not a UT variation. With amplification of the maximal intensity of the equatorial electrojet, its latitudinal extent slightly increases also. At the same time the equatorial electrojet in its maxima of intensity has approximately an identical width, and in the minima the electrojet is narrow in the morning and wide in afternoon. The comparison of experimental data with our calculation results has shown a satisfactory agreement in intensity, position and spatial size of the equatorial electrojet. A small displacement of the maximal intensity of the electrojet into the morning sector in comparison with experimental data, can be connected with the absence of thermospheric tides on the bottom boundary of the thermosphere and the absence of electric fields of magnetospheric convection in our calculations. The presence of the counter electrojet in the evening sector of local time in our calculations which is absent in experimental data, can possibly be explained by the absence of thermospheric tides in our calculations. The UT-variation of the surface density of the equatorial electrojet is much weaker and equals $\sim 1-3 \mathrm{~A} / \mathrm{km}^{2}$ and herewith the maximal intensity is $\sim 15-20 \mathrm{~A} / \mathrm{km}^{2}$. The latitudinal extent of the surface density of the equatorial electrojet is maximal 
at 23:00 UT and 15:00 UT and minimal at 06:00 UT and 20:00 UT.

Acknowledgements. The authors express sincere gratitude to M. Förster for useful discussions and for the presentation of our reports at the. Kleinheubacher Tagung of the URSI Landesausschuss Deutschland e.V., 2006, Miltenberg, Germany, 25-29 September 2006, thanks to which the opportunity of this publication was given.

\section{References}

Baker, W. G. and Martyn, D. F.: Electric Currents in the Ionosphere, I The Conductivity, Phil. Trans. Roy. Soc., London, A246, 281294, 1953.

Chapman, S.: The equatorial electrojet as detected from the abnormal electric current distributions above Huancayo, Peru and elsewhere, Arch. Meteorol. Geophys. Bioklimatol, A4, 368-390, 1951.

Cowling, T. G.: The magnetic field of sunspots, Mon. Not. R. Astron. Soc., 94, 39-48, 1933.

Doumouya, V. and Cohen, V.: Improving and testing the empirical equatorial electrojet model with CHAMP satellite data, Ann. Geophys., 22. 3323-3333, 2004.

Egedal, J.: The magnetic diurnal variation of the horizontal force near the magnetic equator, Terr. Magn. Atmos. Electr., 52, 449451, 1947.

Gershman, B. N.: Dynamics of ionospheric plasmas, Moscow, Nauka, 256p., 1974 (In Russian).

Gurevich, A. V., Krylov, A. L., and Tsedilina, E. E.: Electric Fields in the Earth's Magnetosphere and Ionosphere, Space Sci. Rev., 19, 59-160, 1976.

Heelis, R. A.: Electrodynamics in the low and middle latitude ionosphere: a tutorial, J. Atmos. Solar-Terr. Phys., 66, 825-838, 2004.

Ivers, D, Stening, R., Turner, J., et al.: Equatorial electrojet from Ørsted scalar magnetic field observations, J. Geophys. Res., 108, 1061, doi:10.1029/2002JA009310, 2003.

Klimenko, M. V., Klimenko, V. V., and Bruykhanov, V. V.: Comparison of two variants of model of the electric field in the ionosphere of the Earth, KSTU News, 8, 59-68, 2005 (In Russian).

Klimenko, M. V., Klimenko, V. V., and Bruykhanov, V. V.: Numerical Simulation of the Electric Field and Zonal Current in the Earth's Ionosphere: The Dynamo Field and Equatorial Electrojet, Geomagnetism and Aeronomy, 46, 457-466, 2006 a.
Klimenko, V. V., Klimenko, M. V., and Bruykhanov, V. V.: Numerical modeling of electric field and zonal current in the Earth's ionosphere - Statement of the problem and test calculations, Matematicheskoye Modelirovaniye, 18, 77-92, 2006b (In Russian).

Korenkov, Yu. N., Klimenko, V. V., Forster, M., et al.: Calculated and observed ionospheric parameters for a Magion 2 passage and EISCAT data on June 31, 1990, J. Geophys. Res., 103, $14697-$ 14710, 1998.

Le Mouel, J.-L., Shebalin, P., and Chuliat, A.: The field of the equatorial electrojet from CHAMP data, Ann. Geophys., 24, 515527, 2006, http://www.ann-geophys.net/24/515/2006/.

Lühr, H., Maus, S., and Rother, M.: Noone-time equatorial electrojet: Its spatial features as determined by the CHAMP satellite, J. Geophys. Res., 109, A01306, doi:10.1029/2002JA009656, 2004.

Manoj, C., Lühr, H., Maus, S., et al.: Evidence for short spatial correlation lengths of the noontime equatorial electrojet inferred from a comparison of satellite and ground magnetic data, J. Geophys. Res., 111, A11312, doi:10.1029/2006JA011855, 2006.

Martyn, D. F., Cowling, T.G., and Borger, R.: Electric conductivity of the ionospheric D-region, Nature, Lond., 162, 142-143, 1948.

Namgaladze, A. A., Korenkov, Yu. N., Klimenko, V. V., et al.: Global Model of the Thermosphere-Ionosphere-Protonosphere System, Pure and Applied Geophysics (PAGEOPH), 127, 219254, 1988.

Namgaladze, A. A., Korenkov, Yu. N., Klimenko, V. V., et al.: Numerical modelling of the thermosphere-ionosphereprotonosphere system, J. Atmos. Terr. Phys., 53, 1113-1124, 1991.

Richmond, A.D.: Modeling the ionosphere wind dynamo: A review, Pure and Appl. Geophys. (PAGEOPH), 131, 413-435, 1989.

Richmond, A. D.: Thermospheric Dynamics and Electrodynamics, in Sol.-Terr. Phys., Principles and Theoretical Foundations, edited by: Carovillano, R. L. and Forbes, J. M., D. Reidel Publishing Company, Dordrecht, Holland, 523-607, 1982.

Singh, A., and Cole, K.D.: A Numerical Model of the Ionospheric Dynamo I. Formulation and Numerical Technique, J. Atoms. Terr. Phys., 49, 521-527, 1987.

Stening, R.J.: Modeling the equatorial electrojet, J. Geopys. Res., 90, 1705-1719, 1985.

Sugiura, M. and Cain, J. G.: A Model Equatorial Electrojet, J. Geophys. Res., 71, 1869-1877, 1966.

Volland, H.: Atmospheric Electrodynamics, Springer-Verlag, Berlin, 1984. 